\title{
Thrombosis
}

\section{Platelet-Leukocyte Interactions in Inflammation and Thrombosis}

\author{
Noemi Michetti, PhD, Andrew S Weyrich, PhD and Guy A Zimmerman, MD
}

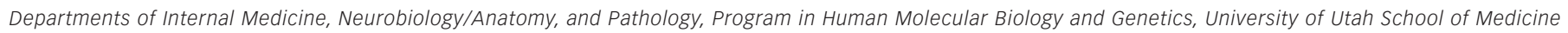

\begin{abstract}
Interactions between human platelets and leukocytes occur in physiological and pathological inflammation, hemostasis, and thrombosis. There is evidence that platelet-leukocyte interactions may be central mechanisms in atherosclerosis and its complications, and also in a variety of other acute and chronic diseases. In addition, models based on interactions of platelets and leukocytes are informative discovery tools that have yielded new insights relevant to human cell functions and cell-cell signaling and information transfer. This short article focuses on interactions between platelets and myeloid leukocytes and profiles some of the key pathways, mechanisms, and outcomes in these cellular dialogues.
\end{abstract}

\section{Keywords}

Platelets, leukocytes, monocytes, neutrophils, P-selectin, integrins, signaling, inflammation, hemostasis, thrombosis, atherosclerosis

Disclosure: Noemi Michetti, PhD, has no conflicts of interest to declare. Andrew S Weyrich, PhD, and Guy A Zimmerman, MD, have sponsored research agreements with BoehringerIngelheim and Novo Nordisk.

Received: February 12, 2009 Accepted: June 19, 2009 DOI: 10.17925/OHR.2009.02.0.24

Correspondence: Guy A Zimmerman, MD, Eccles Institute of Human Genetics, Room 4220, University of Utah School of Medicine, Salt Lake City, UT 84112.

E: guy.zimmerman@hmbg.utah.edu

\section{Intercellular Cross-talk Between Platelets and Leukocytes in Inflamation and Thrombosis}

Platelets circulating in the blood become activated and adhesive at sites of vascular injury and inflammation. Activated platelets can then adhere to one another, a process known as 'aggregation' but also called 'homotypic aggregation,' or adhere to exposed subendothelial matrix. These events have been intensely studied because of their requisite contributions to physiological hemostasis and their roles in pathological thrombosis. ${ }^{1}$ In addition, there is recent evidence, largely from murine models, indicating that platelets can adhere to stimulated or 'inflamed' endothelial cells, ${ }^{2}$ although inhibitory substances such as prostacyclin and nitric oxide released from activated endothelium were previously thought to effectively prevent adhesive interactions between endothelial cells and platelets. ${ }^{3}$ Activated platelets can also adhere to leukocytes of a variety of classes, which is an inter-cellular interaction that in some cases involves 'heterotypic aggregation' and that can occur locally or systemically.

This article will focus on platelet-leukocyte interactions and on the molecular events and functional consequences that are involved. Due to space limitations it is not comprehensive, but instead highlights important observations and concepts from both the recent and earlier literature. Platelet-leukocyte interactions in hemostasis and inflammation have also recently been reviewed in other publications. ${ }^{2,4-7}$

\section{Observations Using Human Platelets, Monocytes, and Polymorphonuclear Leukocytes}

Platelets store, synthesize, and release a number of factors involved in inflammatory events. ${ }^{2,5,8}$ In the most well-known mechanism, platelets translocate factors stored in subcellular granules to the plasma membrane or release them into the circulation. Factors released into solution can activate leukocytes and other 'target' cells in a paracrine fashion (see Figure 1). Although release of stored chemokines and signaling factors is traditionally invoked, platelets also have active synthetic pathways that can contribute paracrine signaling molecules., ${ }^{5,8}$ In addition, molecules retained on the platelet surface can mediate direct intercellular signaling without being released: so-called juxtacrine signaling. ${ }^{9}$ Once expressed on the membrane, some factors are shed by cleavage ${ }^{10}$ and/or are released into the milieu associated with platelet-derived microparticles, ${ }^{11}$ and are then able to alter functions of target cells.

In 1986, Jungi et al. reported that thrombin stimulation of platelets induces translocation and expression of surface proteins, which interact with ligands ('counter-receptors') on monocytes and polymorphonuclear (PMN) leukocytes (neutrophils), resulting in intercellular adhesion. The binding was influenced by temperature and was dependent on extracellular $\mathrm{Ca}^{2+}{ }^{12}$ Many subsequent reports have confirmed this observation and mechanisms that are involved have been identified. Pselectin (CD62P, previously known as GMP-140 and PADGEM), a member of the selectin family of adhesion molecules, is of central importance in adhesive interactions between platelets and leukocytes. ${ }^{13}$ P-selectin is 
stored in $\alpha$-granules in quiescent circulating platelets and is translocated to the plasma membrane upon activation. P-selectin glycoprotein ligand1 (PSGL-1) is a specific ligand for P- and L-selectins, and is expressed on monocytes and PMNs. ${ }^{13}$ Binding of P-selectin to PSGL-1 mediates platelet adhesion to neutrophils and monocytes. ${ }^{14-16}$ Proteinase-activated receptor (PAR) agonists such as thrombin, thrombin-receptor-inhibiting peptide (TRAP), PAR1AP, and PAR4AP induce the formation of platelet-leukocyte aggregates by stimulating P-selectin exposure on the surface of activated platelets and microparticles ${ }^{16-18}$ P-selectin-mediated adhesion of platelets to myeloid leukocytes triggered in this fashion is responsible for stable and strong interactions. Other agonists also induce P-selectin display on activated platelets with variable potency compared with PAR agonists. Monocytes bind P-selectin-expressing platelets more rapidly than PMNs, and platelet-monocyte aggregates form even when weaker platelet agonists are used. ${ }^{15}$ Activated platelets bind not only monocytes and neutrophils but also eosinophils, basophils, and subpopulations of T cells. Like platelet-monocyte and platelet-PMN aggregates, such aggregates are detectable by flow cytometry. ${ }^{19,20}$ P-selectin on the surface of activated platelets also mediates these adhesive interactions. ${ }^{13}$

In addition to forming heterotypic aggregates in solution, leukocytes adhere to immobilized, activated platelets under shear stress conditions intended to model blood flow. Adhesion of PMNs under these conditions is reduced using antibodies against $P$-selectin or ethylenediaminetetraacetic acid (EDTA) to chelate extracellular $\mathrm{Ca}^{2+}$, which is required for binding of P-selectin to PSGL-1. ${ }^{21}$ Platelet-activating factor (PAF) translocated to the surface of collagen-adherent platelets mediates neutrophil activation and firm adhesion to platelet monolayers. ${ }^{9}$ Additional adhesive and signaling mechanisms are also involved. ${ }^{2,7}$

In addition to P-selectin/PSGL-1, other molecular interactions mediate intercellular interactions between activated platelets and myeloid leukocytes (see Figure 1). For example, soluble agonists and P-selectinPSGL-1 binding can alter the activation state of a $\beta_{2}$ integrin, $\alpha_{M} \beta_{2}(C D 11 b /$ CD18, Mac1), on human myeloid leukocytes. ${ }^{22}$ Fibrinogen can then be bound by activated $\alpha_{M} \beta_{2}$ on PMNs and monocytes and also by integrin $\alpha_{\| b} \beta_{3}$ (GPIIb/IIla) on the activated platelets, thereby mediating adhesion of the two cell types ${ }^{2,23-25}$ (see Figure 1). This mechanism supports firm adhesion between platelets and leukocytes. ${ }^{26} \mathrm{As}$ a second example, GPIb $\alpha$ and junctional adhesion molecule 3 (JAM-3) on the platelet plasma membrane bind directly to $\alpha_{\mathrm{M}} \beta_{2}$ displayed by myeloid leukocytes. ${ }^{27,28}$ Recently, it was reported that microparticles bearing activated $\alpha_{M} \beta_{2}$ are released by PMNs and can activate resting platelets by engaging GPIb $\alpha$; this adhesion and signaling event acted in concert with P-selectin-PSGL-1 binding.$^{29}$ Thus, platelet-leukocyte interactions can be bi-directional. ${ }^{30}$

Platelets constitutively express intercellular adhesion molecule-2 (ICAM-2) but not ICAM-1 or -3 on their surfaces. ${ }^{31}$ Although the level of expression of ICAM-2 on the plasma membrane of platelets does not change with activation, it serves as a counter-receptor for another member of the leukocyte $\beta_{2}$ integrin family, $\alpha_{\llcorner} \beta_{2}$ (leukocyte function-associated antigen-1 [LFA-1]), which can mediate adhesion of activated leukocyte subsets to platelets. $^{31}$ Thrombospondin (TSP) is a platelet $\alpha$-granule protein that, similar to P-selectin, is translocated to the platelet membrane upon activation; it is also an antibody against TSP-inhibited platelet-monocyte aggregate formation. ${ }^{32}$ Thus, additional molecular pathways besides P-

\section{Figure 1: Platelet-Leukocyte Interactions Mediate Intercellular Adhesion and Signaling}

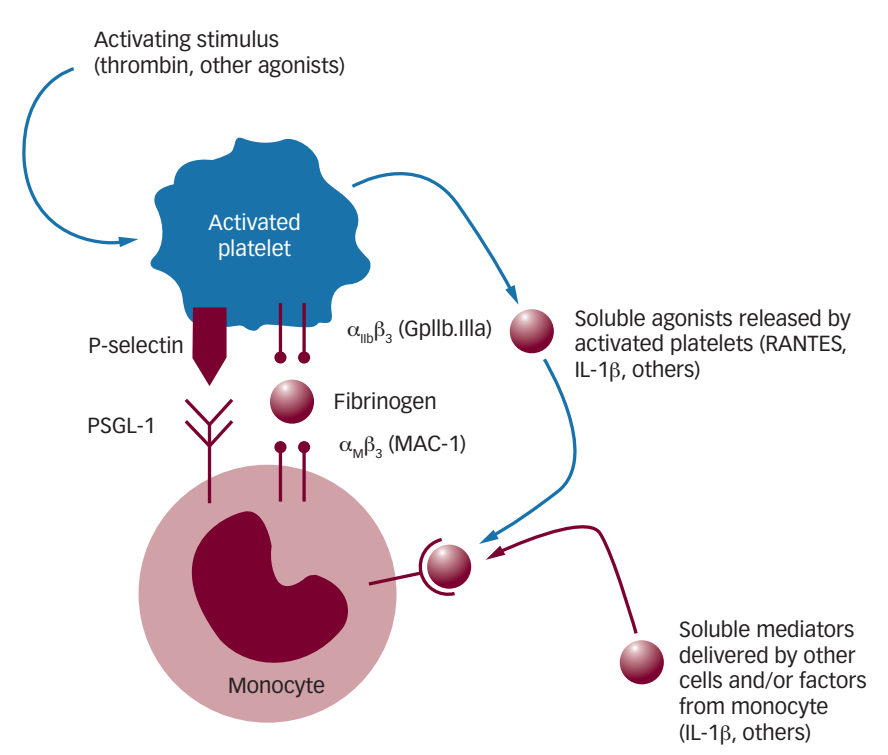

Platelets interact with a variety of myeloid leukocytes and other leukocyte subtypes. A platelet interacting with a monocyte is shown in this figure to illustrate major concepts. Activation of human platelets with thrombin or other agonists induces translocation of P-selectin from intracellular alpha granules to the cell surface. P-selectin displayed on the plasma membrane of the platelet can then bind to P-selectin glycoprotein ligand-1 (PSGL-1), which is constitutively present on the monocyte surface, mediating stable cell-cell adhesion and transmitting 'outside-in' signals to intracellular transduction pathways in the leukocyte. Adhesion mediated by integrin $\alpha / 1 / b 3$ on the activated platelet and integrin $\alpha \beta_{32}$ on the monocyte with engagement of soluble fibrinogen by both integrins can also contribute to adhesion and signaling. Factors such as RANTES and interleukin (IL)-1 $\beta$ that are released from the activated platelet can deliver signals to the monocyte in parallel with signaling by adhesion molecules, inducing or amplifying intracellular signaling and consequent functional alterations in leukocytes. Expression of new inflammatory proteins mediated by transcriptional and posttranscriptional pathways activated by intercellular signaling is an example of one such functional alteration. Signaling from the monocyte to the platelet may also occur, although this has not been as extensively studied. In addition to the molecular events illustrated here, other adhesive and signaling interactions between human and/or murine platelets and myeloid leukocytes have also been reported. These involve members of the platelet glycoprotein, leukocyte $\left(\beta_{2}\right)$ integrin, inter-cellular adhesion molecule 1 (ICAM), and junctional adhesion molecule (JAM) families.

selectin-PSGL-1, integrins, ICAMs, and platelet glycoproteins mediate membrane contact and interactions between platelets and leukocytes. As noted above, many factors such as chemokine (C-C) ligand 5 (CCL5, also known as RANTES), soluble CD40 ligand (SCD40L), matrix metalloproteinases (MMPS), platelet factor 4 (PF4), and interleukin $1 \beta(\mathrm{IL}-1 \beta)$ are released into the milieu by activated platelets and can provide signals to leukocytes without direct cell-cell interaction. ${ }^{2,5}$ Nevertheless, these paracrine factors and/or soluble factors released by leukocytes themselves or other cell types can act in concert with signals delivered by P-selectin-PSGL-1, integrins, or other adhesion ligands, providing a mechanism for signal integration and amplification ${ }^{16,33}$ (see Figure 1).

\section{Platelet-Leukocyte Interactions- Animal Models}

In vivo animal models have been used to study platelet-leukocyte interactions in inflammatory and thrombotic events. Apolipoprotein E-deficient $\left(\mathrm{ApoE}^{-/-}\right)$hypercholesterolemic mice are commonly employed to examine development of atherosclerosis. ${ }^{34,35}$ Recently, it was reported that these animals are characterized by early adhesion of platelets to the endothelium, resulting in recruitment of leukocytes to the carotid artery, suggesting that this is critical in the initiation of atherosclerotic 


\section{Thrombosis}

\section{Table 1: Clinical Conditions in Which Platelet-Leukocyte Interactions Have Been Reported or Platelet-Leukocyte Aggregates Have Been Detected in the Circulation}

\begin{tabular}{ll} 
Atherosclerosis & Sepsis \\
\hline Acute coronary syndromes & Acute lung injury \\
\hline Percutaneous coronary interventions & Cystic fibrosis \\
\hline Cerebrovascular ischemia and stroke & Complications of transfusion \\
\hline Venous stasis ulceration & Hip arthroplasty \\
\hline Smoking & Ulcerative colitis \\
\hline Diabetes & Cirrhosis \\
\hline
\end{tabular}

Iesion development. ${ }^{36}$ Furthermore, animal studies indicated that platelet-leukocyte aggregates form and associate with endothelium in response to cigarette smoke, a known environmental risk factor for atherosclerosis. ${ }^{37}$

In models of vessel injury, P-selectin-deficient mice accumulate fewer leukocytes, resulting in protection against intimal hyperplasia and reduced number and size of atherosclerotic lesions. ${ }^{38,39} \mathrm{ApoE}^{-/-}$mice transplanted with bone marrow from P-selectin $/$ - mice had 30\% smaller atherosclerotic lesions than $\mathrm{ApoE}^{-/-}$mice transplanted with P-selectin+/+ marrow; this was interpreted as indicating a role for platelet-expressed P-selectin in atherosclerosis lesions. ${ }^{40}$ Consistent with this possibility, one potential explanation is adhesion of activated platelets to myeloid leukocytes and recruitment of these cells to the evolving atherosclerotic plaques. In a different study, infusion of activated platelets into C57BL/6 mice increased the number of circulating leukocyte-platelet aggregates. This induced monocyte and, to a lesser extent, neutrophil accumulation in the atherosclerotic lesion, indicating that circulating activated platelets and platelet-leukocyte aggregates promote the evolution of atherosclerotic lesions. ${ }^{41}$ Intercellular signaling between platelets and myeloid leukocytes in vivo may be required for their local accumulation in experimental vascular injury. ${ }^{42}$ Such adhesion and signaling interactions occur in both experimental and clinical angioplasty and vascular stent placement. ${ }^{43,44}$

Animal models also suggest important roles for platelet interactions with leukocytes in other inflammatory syndromes besides atherosclerosis and its sequelae. For example, several studies indicate that this is a feature of murine models of acute lung injury and experimental acute respiratory distress syndrome. ${ }^{7}$ Nevertheless, it should be remembered that there are differences in circulating platelet and leukocyte numbers and perhaps function in mice and other species, ${ }^{7,45}$ which may influence the clinical relevance of outcomes in these experimental systems.

\section{Functional Consequences of Platelet-Leukocyte Interactions in Inflammation and Thrombosis- Intercellular Signaling and Signal Transduction}

Platelet-leukocyte interactions appear to be mechanisms for targeting and local accumulation of leukocytes in physiological inflammation and hemostasis, and for local or systemic cell-cell contact in pathological inflammatory and thrombotic syndromes. ${ }^{2,718}$ It has also been suggested that formation of platelet-leukocyte aggregates contributes to the clearance of activated cells from the circulating blood. ${ }^{4}$ In pathological conditions in which platelet-monocyte or platelet-neutrophil aggregates form and are retained in the vasculature, these multicell clusters may be involved in mechanical occlusion of vessels, such as coronary arteries ${ }^{4}$ or lung capillaries.7 However, in addition to mechanical consequences, platelet-leukocyte interactions can alter the functional responses of both the leukocyte and the platelet. ${ }^{30}$ For example, platelet activation resulting from signals delivered by myeloid leukocytes or leukocyte microparticles may amplify thrombosis by triggering $\alpha_{\| b} \beta_{3}$-dependent adhesive events and surface expression of P-selection. ${ }^{29}$ P-selectin is reported to act synergistically with tissue factor, which is generated in platelet-monocyte interactions and by activated platelets, ${ }^{46-49}$ thereby accelerating fibrin deposition. ${ }^{4}$ Conversely, it is clear that signals delivered to monocytes or PMNs by activated platelets can induce functional responses by the leukocytes that have inflammatory and thrombotic consequences. Some of these signaling mechanisms have been characterized relatively recently, while others are still in the process of being identified and dissected.

Signals from activated platelets induce expression of nuclear factor kappa $\mathrm{B}$ (NF-kB)-dependent genes by human monocytes. An early report described synthesis of monocyte chemotactic protein 1 (MCP-1) by human monocytes adherent to thrombin-stimulated platelets in heterotypic clusters. ${ }^{16}$ The mechanism involves parallel signaling via P-selectin-PSGL-1 engagement and the soluble chemokine RANTES, which is released from activated platelets and is recognized by CC chemokine receptor 5 (CCR5) on the monocyte (see Figure 1); these signals are integrated to yield transcription of the messenger RNA (mRNA) for MCP-1 and synthesis of the protein. ${ }^{16}$ Monocytes also synthesized IL-8 and tumor necrosis factor alpha (TNF- $\alpha$ ) under these conditions.

More recently, engagement of PSGL-1 on human monocytes by P-selectin and parallel signaling by IL-1 $\beta$ were shown to mediate biphasic and temporally regulated expression of cyclo-oxygenase 2 (cox-2) in adhesive interactions of the leukocytes with thrombin-stimulated platelets. ${ }^{33}$ Apart from thrombin, additional agonists trigger formation of human platelet-monocyte aggregates and consequent chemokine synthesis. ${ }^{37}$ Early and more recent studies using primary monocytes and myeloid cells transfected with reporter constructs demonstrated that engagement of PSGL-1 induces nuclear translocation and activation of $\mathrm{NF}-\kappa \mathrm{B}$; parallel signaling by RANTES or IL-1 can then deliver parallel signals for transcriptionally controlled expression of multiple NF-кB-dependent inflammatory gene products. ${ }^{16,33}$ Nevertheless, there are additional pathways in the repertoire of mechanisms by which the pattern of genes expressed by monocytes can be altered by intercellular signaling in platelet-leukocyte interactions. There is also evidence that engagement of PSGL-1 by P-selectin signals to specialized translational control checkpoints. ${ }^{50,51}$ For example, adhesion of human monocytes to purified, immobilized P-selectin or to activated platelets in heterotypic aggregates induces expression of urokinase plasminogen activator receptor (UPAR), a surface protease receptor and regulator of cell adhesion and migration..$^{50}$ Engagement of PSGL-1 appears to be sufficient for rapid synthesis of UPAR from constitutive, silenced mRNA without a requirement for new transcription, although transcription also occurs. This specialized translational mechanism was shown to be mediated by mammalian target of rapamycin (mTOR), identifying a new role for the complex pathway regulated by this kinase in myeloid leukocytes. ${ }^{50}$ Additional post-transcriptional pathways in monocytes may be triggered by interactions with activated platelets, ${ }^{51}$ and the studies together indicate that the pattern of inflammatory proteins expressed by the leukocytes in response to signals from platelets is regulated by precise 
transcriptional and post-transcriptional mechanisms in a gene-specific and temporally controlled fashion..$^{16,3,50}$ Specific pathways may thus be targets for therapeutic intervention in pathological inflammation involving platelet-monocyte signaling. ${ }^{52}$

Similar functional responses also occur in models in which PMNs interact with activated platelets, although cell-specific variations undoubtedly occur. For example, earlier and more recent observations indicated that engagement of PSGL-1 on human PMNs activates mitogen-activated protein (MAP) kinases, ${ }^{53}$ and that PSGL-1 in parallel with the PAF receptor can signal to components of the mTOR pathway ${ }^{54}$ in human neutrophils. Thus, intracellular transduction cascades in PMNs are activated by signals from platelets. Taken together, the studies also suggest that platelet-PMN interactions may induce synthesis of chemokines and other inflammatory factors by PMNs when platelet-neutrophil aggregates form or PMNs adhere to platelets at sites of vascular injury. The findings further suggest that in PMNs, as with monocytes, both transcriptional and post-transcriptional pathways are triggered by signals from activated platelets. Conversely, it is unknown whether signaling by adherent PMNS or monocytes induces post-transcriptional expression of new proteins by the interacting platelets, mediated by transduction pathways that have recently been described. ${ }^{8}$ Nevertheless, the evidence for altered gene expression and synthesis of inflammatory and hemostatic proteins in platelet-leukocyte interactions that we have briefly reviewed here adds to the complexity of synthetic responses, including production of biologically active lipid mediators and other factors, ${ }^{30,55}$ that have been previously recognized in molecular dialogs between these cell types.

Platelet signaling of leukocytes may induce additional intricate responses relevant to inflammation and thrombosis. Several reports indicate that interactions of monocytes with platelets in vitro alter cell fate determination as the leukocytes are induced to differentiate toward macrophages or dendritic cell lineage end-points. ${ }^{5,56,57}$ Thus, platelet-leukocyte interactions have the potential to broadly influence inflammatory responses by altering the developmental pathways of specialized leukocytes that are major regulators of innate and acquired immune functions and that mediate interplay between coagulation and inflammation. ${ }^{5,7,58}$

\section{Clinical Implications}

Table 1 lists some of the clinical conditions in which circulating platelet-leukocyte aggregates, usually heterotypic complexes of platelets and monocytes or PMNs, have been detected and/or platelet-leukocyte interactions have been implicated in the pathogenesis of the syndrome or condition. These observations suggest that complex interactions between human platelets and leukocyte subsets are fundamental mechanisms of inflammation and thrombosis in a large cross-section of human diseases. Additional investigations that will address this topic in addition to contribution of platelet-leukocyte interactions to specific diseases and pathological conditions are in progress.

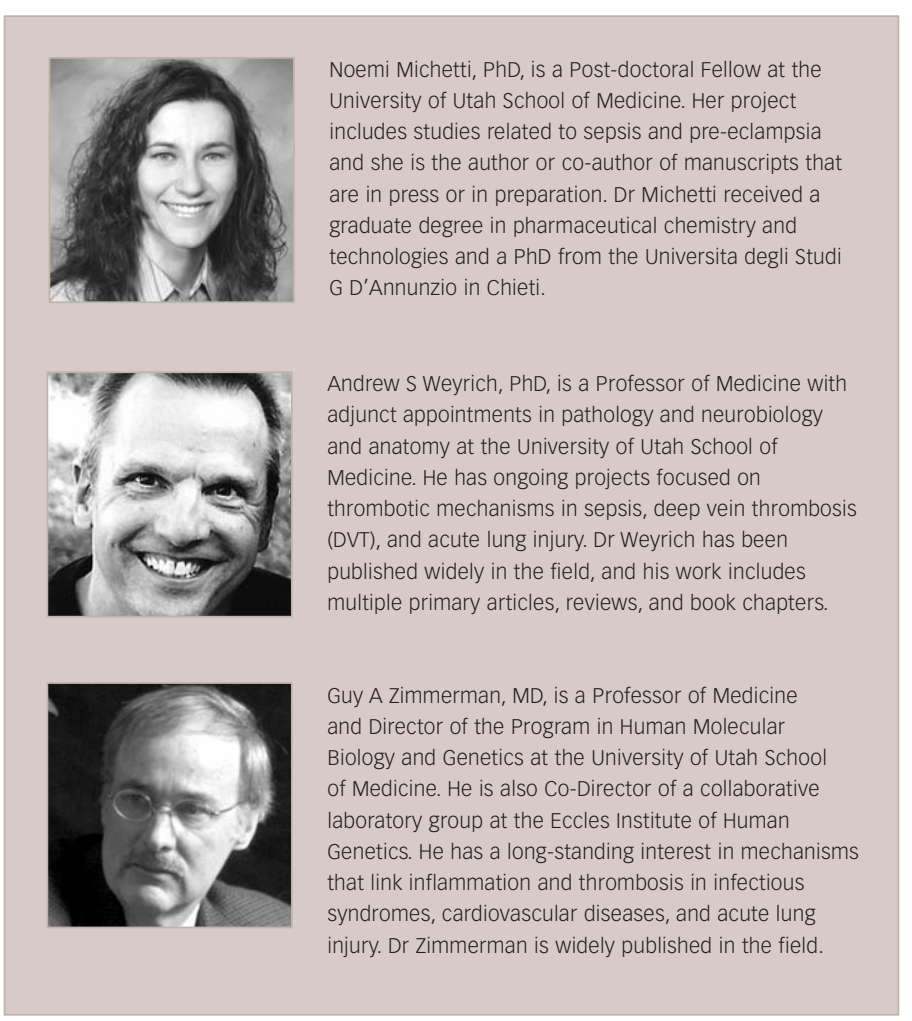

1. Davi G, N Eng/ J Med, 2007;357:2482-94.

2. Bergmeier $W$, Wagner $D D$, In: Michaelson $A D(e d)$, Platelets, Boston: Accademy Press, 2007;713-26.

3. Roth GJ, Immunol Today, 1992;13:100-105.

4. Freedman JE, Loscalzo J, Circulation, 2002; 105:2130-32.

5. Weyrich AS, Zimmerman GA, Trends Immunol, 2004;25:489-95.

6. Gawaz M, et al., J Clin Invest, 2005;115:3378-84

7. Bozza FA, et al., Am J Respir Cell Mol Biol, 2009;40:123-34.

8. Zimmerman GA, Weyrich AS, Arterioscler Thromb Vasc Biol, 2008; 28:s17-24.

9. Ostrovsky L, et al., Blood, 1998;91:3028-36.

10. Freedman JE, Circ Res, 2003;92:944-6.

11. Reininger AJ, et al., Blood, 2006;107:3537-45.

12. Jungi TW, et al., Blood, 1986;67:629-36.

13. McEver RP, Thromb Haemost, 2001;86:746-56.

14. Hamburger SA, McEver RP, Blood, 1990;75:550-54.

15. Rinder HM, et al., Blood, 1991;78:1730-37.

16. Weyrich AS, et al., J Clin Invest, 1996;97:1525-34

17. Chung AW, et al., Br J Pharmacol, 2004;143:845-55.

18. Weyrich AS, J Thromb Haemost, 2003;1:1897-1905.

19. de Bruijne-Admiraal LG, et al., Blood, 1992;80:134-42.

20. Michelson AD, et al., Circulation, 2001;104:1533-7.

21. Buttrum SM, Hatton R, Nash GB, Blood, 1993;82:1165-74.
22. Lorant $\mathrm{DE}$, et al., J Clin Invest, 1993;92:559-70.

23. Gawaz MP, et al., J Clin Invest, 1991;88:1128-34.

24. Neumann FJ, et al., J Am Coll Cardiol, 1999; 34:1420-26.

25. Peters MJ, et al., J Immunol Methods, 1997;209:125-35.

26. Spangenberg P, et al., Thromb Haemost, 1993;70:514-21.

27. Simon DI, et al., J Exp Med, 2000;192:193-204.

28. Santoso S, et al., J Exp Med, 2002;196:679-91.

29. Pluskota E, et al., Blood, 2008;112:2327-35.

30. Elstad MR, et al., Curr Opin Hematol, 1995;2:47-54

31. Diacovo TG, et al., J Clin Invest, 1994; 94:1243-51.

32. Silverstein RL, Nachman RL, J Clin Invest, 1987;79:867-74.

33. Dixon DA, et al., J Clin Invest, 2006;116:2727-38.

34. Nakashima $Y$, et al., Arterioscler Thromb, 1994;14: 133-40.

35. Zadelaar S, et al., Arterioscler Thromb Vasc Biol, 2007;27: 1706-21.

36. Massberg S, et al., J Exp Med, 2002;196:887-96.

37. Lehr HA, et al., J Clin Invest, 1997;99:2358-64.

38. Smyth SS, et al., Circulation, 2001;103:2501-7.

39. Dong ZM, Brown AA, Wagner DD, Circulation, 2000;101:2290-95.

40. Burger PC, Wagner DD, Blood, 2003;101:2661-6.

41. Huo Y, et al., Nat Med, 2003:9:61-7.

42. Evangelista V, et al., Blood, 2007;109:2461-9.
43. Palmerini T, et al., J Am Coll Cardiol, 2002;40:360-66.

44. Welt FG, Rogers C, Arterioscler Thromb Vasc Biol, 2002;22: 1769-76.

45. Tsakiris DA, et al., Thromb Haemost, 1999;81:177-88.

46. Lindmark E, Tenno T, Siegbahn A, Arterioscler Thromb Vasc Biol, 2000;20:2322-8

47. Steiner $\mathrm{S}$, et al., Arterioscler Thromb Vasc Biol, 2003;23: 1697-1702.

48. Schwertz H, et al., J Exp Med, 2006:203:2433-40.

49. Brambilla M, et al., Arterioscler Thromb Vasc Biol, 2008;28 947-53.

50. Mahoney TS, et al., Proc Natl Acad Sci U S A, 2001;98: 10284-9.

51. Lindemann SW, et al., Trends Cardiovasc Med, 2005;15:9-17.

52. Weyrich AS, et al., Circulation, 2005;111:633-42.

53. Hidari KI, et al., J Biol Chem, 1997;272:28750-56.

54. Lindemann SW, et al., Proc Natl Acad Sci U S A, 2004;101:7076-81.

55. Elstad MR, et al. J Immunol , 1995:155:2109-22.

56. Ammon C, et al., J Leukoc Biol, 1998;63:469-76.

57. Li G, et al. .J Immunol, 2003:171:669-77.

58. Niessen F, et al., Nature, 2008;452:654-8. 